\title{
Case Report \\ Shedding of the Pandemic Swine-Origin Influenza A Virus (H1N1) after Oseltamivir Administration
}

\author{
Leiyun Weng, ${ }^{1}$ Qiang Wang, ${ }^{1}$ Wei Wang, ${ }^{2}$ Peijun Ren, ${ }^{2}$ Vincent Deubel, ${ }^{2,3}$ \\ and Tetsuya Toyoda $\mathbf{a}^{1,4,5}$ \\ ${ }^{1}$ Unit of Viral Genome Regulation, Institut Pasteur of Shanghai, The Key Laboratory of Virology and Immunology, Chinese Academy \\ of Sciences, 411 Hefei Road, Shanghai 200025, China \\ ${ }^{2}$ Emerging Infectious Diseases, Institut Pasteur of Shanghai, The Key Laboratory of Virology and Immunology, Chinese Academy of \\ Sciences, 411 Hefei Road, Shanghai 200025, China \\ ${ }^{3}$ Institut Pasteur in Cambodia, 5 Monivong Boulevard, P.O. Box 983, Phnom Penh, Cambodia \\ ${ }^{4}$ Choju Medical Institute, Fukushimura Hospital, 19-4 Azanakayama, Noyori-cho, Toyohashi, Aichi 441-8124, Japan \\ ${ }^{5}$ Infectious Disease Regulation Project, Tokyo Metropolitan Institute of Medical Sciences, 1-6, Kamikitazawa 2-chome, \\ Setagaya-ku, Tokyo156-8506, Japan
}

Correspondence should be addressed to Tetsuya Toyoda, toyoda_tetsuya@yahoo.co.jp

Received 1 October 2010; Revised 5 November 2010; Accepted 15 November 2010

Academic Editor: Daniel Roberto Perez

Copyright (C) 2010 Leiyun Weng et al. This is an open access article distributed under the Creative Commons Attribution License, which permits unrestricted use, distribution, and reproduction in any medium, provided the original work is properly cited.

We analyzed the virus shedding of an oseltamivir-treated patient who had been infected with the pandemic swine-origin influenza A (H1N1) virus which had an oseltamivir-sensitive neuraminidase. The virus was isolated from the pharyngeal swabs of the patient using MDCK cells, and the virus genome RNA was detected in the same samples both by real-time RT-PCR and RTPCR. The virus was isolated until $44 \mathrm{~h}$ after oseltamivir administration although the virus genome was detected until one day after oseltamivir treatment was stopped. Due to their high sensitivity, RT-PCR and real-time RT-PCR may cause misdiagnosis by detection of viral genome which does not infect, and classical virus isolation and clinical symptoms are recommended for the evaluation of oseltamivir treatment.

\section{Introduction}

The novel swine origin influenza A virus (S-OIV) (H1N1) caused pandemic in 2009-2010 [1]. Oseltamivir (Tamiflu, Hoffmann LaRoche) is recommended for the treatment of S-OIV infection because it is naturally resistant to amantadine and rimantadine but is susceptible to the neuraminidase inhibitors, oseltamivir, and zanamivir (Relenza, Glaxo Smithkline) although the oseltamivir-resistant neuraminidase (NA) H275Y (N1 numbering) was detected in some isolates [2-4]. Here, we precisely report the virus shedding of the pandemic S-OIV after oseltamivir treatment (Table 1).

\section{Case Presentation}

A 24-year-old female in Shanghai, China was infected with the pandemic S-OIV in September 2009. She had received a seasonal flu vaccination (Sanofi Pasteur) the previous year.

Her first symptom was a sore throat, and on the 4th day after the onset of her sore throat, she had a high fever $\left(>38.5^{\circ} \mathrm{C}\right)$. The next day (the 5th day after onset), she visited a clinic. Her blood cell count was normal. Her chest X-ray did not show bronchitis or pneumonia. Oseltamivir $(75 \mathrm{mg} \times 2)$ was administered at 36 hours after the onset of her high fever for five days. On the second day of oseltamivir administration, her high fever disappeared, and all eight segments of the S-OIV genome were cloned from her pharyngeal swabs, and the sequences of the pandemic S-OIV were confirmed. Its neuraminidase (NA) contained an oseltamivir-sensitive sequence $(275 \mathrm{H})$. Since then, pharyngeal swabs were collected everyday for eight days and subjected to virus isolation and examined for virus genome RNA (from the 6th to the 13th day).

Each pharyngeal swab was aliquoted into three fractions: for virus isolation, for RT-PCR, and for real-time RT-PCR, 
TABLe 1: Symptoms and detection of S-OIV.

\begin{tabular}{|c|c|c|c|c|c|c|c|c|c|c|c|c|c|}
\hline Date (2009) & Sep 16 & 17 & 18 & 19 & 20 & 21 & 22 & 23 & 24 & 25 & 26 & 27 & 28 \\
\hline Time (day) & 1 & 2 & 3 & 4 & 5 & 6 & 7 & 8 & 9 & 10 & 11 & 12 & 13 \\
\hline Oseltamivir & & & & & $++^{1}$ & ++ & ++ & ++ & ++ & + & & & \\
\hline Real-time RT-PCR & & & & & & +++ & ++ & + & + & + & + & - & - \\
\hline RT-PCR & & & & & & + & + & + & + & + & + & - & - \\
\hline Virus isolation & & & & & & $t^{2}$ & $+^{3}$ & - & - & - & - & - & - \\
\hline Fever $\left(>38.5^{\circ} \mathrm{C}\right)$ & & & - & + & + & - & - & - & - & - & - & - & \\
\hline Sore throat & + & + & + & + & + & +++ & +++ & +++ & ++ & - & - & - & \\
\hline Headache & - & - & + & + & ++ & ++ & +++ & +++ & + & - & - & - & \\
\hline Cough & - & - & + & + & + & +++ & +++ & +++ & ++ & + & \pm & - & \\
\hline
\end{tabular}

${ }^{1}$ Seventy-five mg of oseltamivir. The first tablet was administered around 21:00.

${ }^{2}$ Pharyngeal swab was taken around 17:00 (20 h after oseltamivir administration).

${ }^{3}$ Pharyngeal swab was taken around 17:00 (44 h after oseltamivir administration).

TABLE 2: Sequences used for RT-PCR and real-time RT-PCR.

\begin{tabular}{|c|c|}
\hline Name & Sequence $\left(5^{\prime} \rightarrow 3^{\prime}\right)$ \\
\hline PB2 f (BamHI) & CTGGATCCATGGAGAGAATAAAAGAACTGAGAGATC \\
\hline PB2 r (NotI) & CTGCGGCCGCCTAATTGATGGCCATCCGAATTC \\
\hline PB1 f (BamHI) & CTGGATCCATGGATGTCAATCCGACTCTACTTTTC \\
\hline PB1 r (NotI) & CTGCGGCCGCTTATTTTTGCCGTCTGAGTTCTTC \\
\hline PA f (BamHI) & GCGGATCCATGGAAGACTTTGTGCGACAATG \\
\hline PA r (NotI) & GCGCGGCCGCCTACTTCAGTGCATGTGTGAGGAAGG \\
\hline HA f (NheI) & GCTAGCCATGAAGGCAATACTAGTAGTTCTGC \\
\hline HA r (EcoRI) & GAATTCTTAAATACATATTCTACACTGTAGAGACCC \\
\hline NP f (KpnI) & GCGGTACCATGGCGTCTCAAGGCACCA \\
\hline NP r (PstI) & ATCTGCAGTCAACTGTCATACTCСТCTGCATTGTC \\
\hline NAf & ATGAATCCAAACCAAAAGATAATAACC \\
\hline NA $r$ & TTACTTGTCAATGGTAAATGGCAAC \\
\hline $\mathrm{Mf}$ & ATGAGTCTTCTAACCGAGGTCG \\
\hline $\mathrm{Mr}$ & TTACTCTAGCTCTATGTTGACAAAATG \\
\hline NS $f$ & ATGGACTCCAACACCATGTCAAG \\
\hline NS r & TTAAATAAGCTGAAACGAGAAAGCTCT \\
\hline HA primer 1 & GTAAAGCTGGAATCAACAAGG \\
\hline HA primer 2 & GAGACCCATTAGAGCACATC \\
\hline HA probe & TET-GATTGCCCCCAGGGAGACTA-BHQ1 \\
\hline
\end{tabular}

and each experiment was performed in a different lab. For virus genome detection, RNA was extracted from the pharyngeal swabs using Trizol (Invitrogen). The RNA was then analyzed by real time RT-PCR for S-OIV haemagglutinin (HA), which was performed in a SmartCycler II System (Cepheid) using a QuantiTect Probe RT-PCR kit (Qiagen) with the primers (HA primer 1 and 2) and HA probe listed in Table 2. At the same time, the RNA was analyzed for the eight genome segments by RT-PCR. The cDNA was reverse transcribed by MMLV RTase (Takara) using Uni12 (AGCAAAGCAGG and AGCGAAAGCAGG) [5], before being subjected to PCR using LA-Taq (Takara) for the coding frames of the eight genome segments and the primers listed in Table 2.

Her pharyngeal swabs were also inoculated into MDCK cells after filtration through $0.22 \mu \mathrm{m}$ filter (Millipore) and then incubated in DMEM with $2 \mu \mathrm{g} / \mathrm{ml}$ trypsin (DIFCO),
$1 \mathrm{mg} / \mathrm{ml}$ BSA (Sigma), 50 units/ml of penicillin (Invitrogen), $50 \mu \mathrm{g} / \mathrm{ml}$ of streptomycin (Invitrogen), and $0.1 \mathrm{mg} / \mathrm{ml}$ kanamycin (Invitrogen) at $37^{\circ} \mathrm{C}$ for the detection of cytopathic effects (CPEs). When CPEs were detected, the pandemic S-OIV genome segments were also identified by RTPCR of the culture supernatant.

Oseltamivir is accepted as the standard treatment for SOIV $[2,4]$. Although oseltamivir was effective, the virus was isolated from her pharyngeal swabs on the second (20 hours) and third (44 hours) days of oseltamivir administration. The eight viral genome segments were detected by RT-PCR, and HA genome was detected by real-time RT-PCR until the day after the end of oseltamivir administration. No other people around her showed any symptoms.

By 36 hours of oseltamivir administration in previous cases, the influenza A/Texas/91 (H1N1) virus had disappeared from the patients' nasopharyngeal swabs [6]. 
In this case, the virus (influenza virus A/Shanghai/P1/2009 (H1N1)) was still isolated from the patient's pharyngeal swab on the third day (44 hours) of oseltamivir administration, and the viral genome segments were detected until the day after oseltamivir administration was stopped. RT-PCR detection is more sensitive than virus isolation [7]. The +++ and ++ scores of real-time RT-PCR correlated with those of virus isolation. The prolonged detection of viral RNA without the oseltamivir-resistant mutation of NA by RTPCR or real-time RT-PCR from the S-OIV-infected patients administrated with oseltamivir was also reported previously $[8,9]$. All of these reports indicate that S-OIV could persist in the patients although shedding of S-OIV may vary from case to case. However, due to their high sensitivity, RT-PCR and real-time RT-PCR may cause misdiagnosis, and the classical virus isolation and clinical symptoms are still recommended [10].

\section{Conclusions}

The classical virus isolation and clinical symptoms are recommended for the evaluation of oseltamivir treatment although RT-PCR and real-time RT-PCR are convenient.

\section{Consent}

Written informed consent was obtained from the patient for publication of this case report and any accompanying images. A copy of the written consent is available for review by the Editor-in-Chief of this journal.

\section{Conflict of Interests}

The authors declare that they have no conflict of interests.

\section{Authors' Contributions}

L. Weng and Q. Wang collected samples and performed RT-PCR, W. Wang, P. Ren, and V. Deubel performed virus isolation and real-time RT-PCR, and L. Weng and T. Toyoda wrote the paper.

\section{List of Abbreviations}

WHO: The World Health Organization

S-OIV: The novel swine origin influenza A virus

HA: Haemagglutinin

NA: Neuraminidase

RT-PCR: Reverse transcription polymerase chain reaction

CPE: Cytopathic effect.

\section{Acknowledgments}

This work was supported by Grants-in-Aid from the Chinese Academy of Sciences (no. 0514P51131), National Science Foundation of China (no. 30670090), Li Ka Shing Foundation (no. 0682P11131), the French Agency for Development (SISEA Project; no. 0682P21111), and FLUINNATE (no. SP5B-CT-2006-044161). The GenBank account numbers of the influenza virus A/Shanghai/P1/2009 (H1N1) sequences are AB539736-AB539743. The real-time RT-PCR and RT-PCR protocols are available on request. L. Weng and Q. Wang contributed equally to this work.

\section{References}

[1] WHO, "World now at the start of 2009 influenza pandemic," http://www.who.int/csr/don/2009_10_02/en/index.html.

[2] B. Cao, X. W. Li, YU. Mao et al., "Clinical features of the initial cases of 2009 pandemic influenza A (H1N1) virus infection in China," The New England Journal of Medicine, vol. 361, no. 26, pp. 2507-2517, 2009.

[3] E. van der Vries, M. Jonges, S. Herfst et al., "Evaluation of a rapid molecular algorithm for detection of pandemic influenza A (H1N1) 2009 virus and screening for a key oseltamivir resistance (H275Y) substitution in neuraminidase," Journal of Clinical Virology, vol. 47, no. 1, pp. 3437, 2010

[4] R. J. Hall, M. P. Peacey, J. C. Ralston et al., "Pandemic influenza $\mathrm{A}(\mathrm{H} 1 \mathrm{~N} 1) \mathrm{v}$ viruses currently circulating in New Zealand are sensitive to oseltamivir," Eurosurveillance, vol. 14, no. 30, p. 19282, 2009.

[5] E. Hoffmann, J. Stech, Y. Guan, R. G. Webster, and D. R. Perez, "Universal primer set for the full-length amplification of all influenza A viruses," Archives of Virology, vol. 146, no. 12, pp. 2275-2289, 2001.

[6] F. G. Hayden, J. J. Treanor, R. S. Fritz et al., "Use of the oral neuraminidase inhibitor oseltamivir in experimental human influenza: randomized controlled trials for prevention and treatment," Journal of the American Medical Association, vol. 282, no. 13, pp. 1240-1246, 1999.

[7] S. Leekha, N. L. Zitterkopf, M. J. Espy, T. F. Smith, R. L. Thompson, and P. Sampathkumar, "Duration of influenza A virus shedding in hospitalized patients and implications for infection control," Infection Control and Hospital Epidemiology, vol. 28, no. 9, pp. 1071-1076, 2007.

[8] H. Fleury, S. Burrel, C. Balick Weber et al., "Prolonged shedding of influenza $\mathrm{A}(\mathrm{H} 1 \mathrm{~N} 1) \mathrm{v}$ virus: two case reports from France 2009," Eurosurveillance, vol. 14, no. 49, Article ID 19434, 2009.

[9] L. M. Ling, A. L. Chow, D. C. Lye et al., "Effects of early oseltamivir therapy on viral shedding in 2009 pandemic influenza A (H1N1) virus infection," Clinical Infectious Diseases, vol. 50, no. 7, pp. 963-969, 2010.

[10] G. Boivin, Z. Coulombe, and C. Wat, "Quantification of the influenza virus load by real-time polymerase chain reaction in nasopharyngeal swabs of patients treated with oseltamivir," Journal of Infectious Diseases, vol. 188, no. 4, pp. 578-580, 2003. 


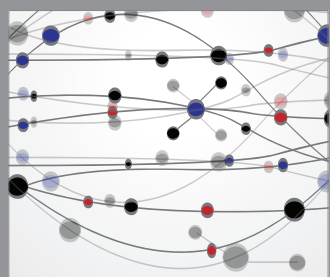

The Scientific World Journal
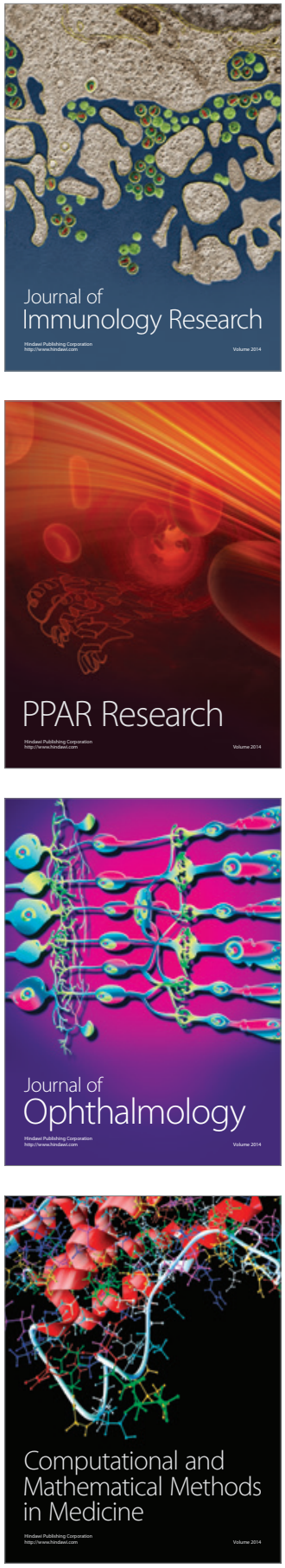

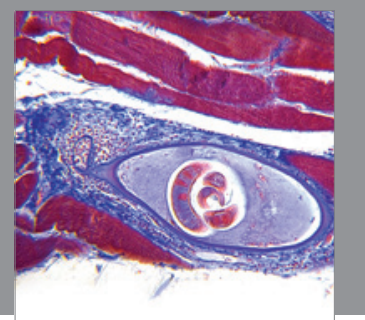

Gastroenterology

Research and Practice
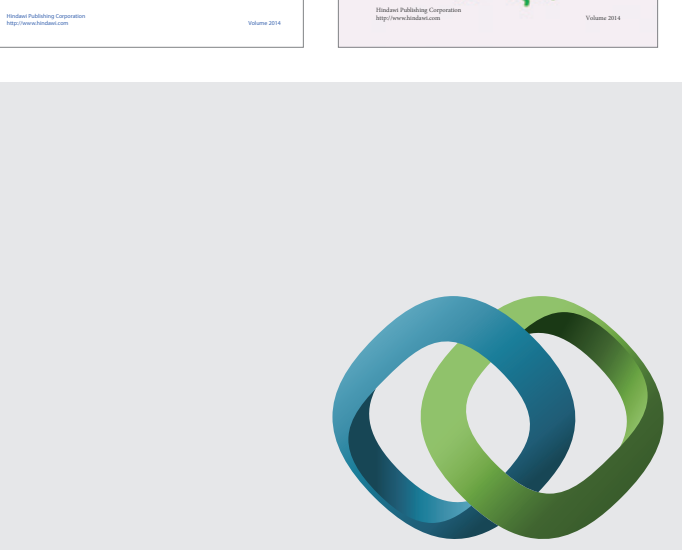

\section{Hindawi}

Submit your manuscripts at

http://www.hindawi.com
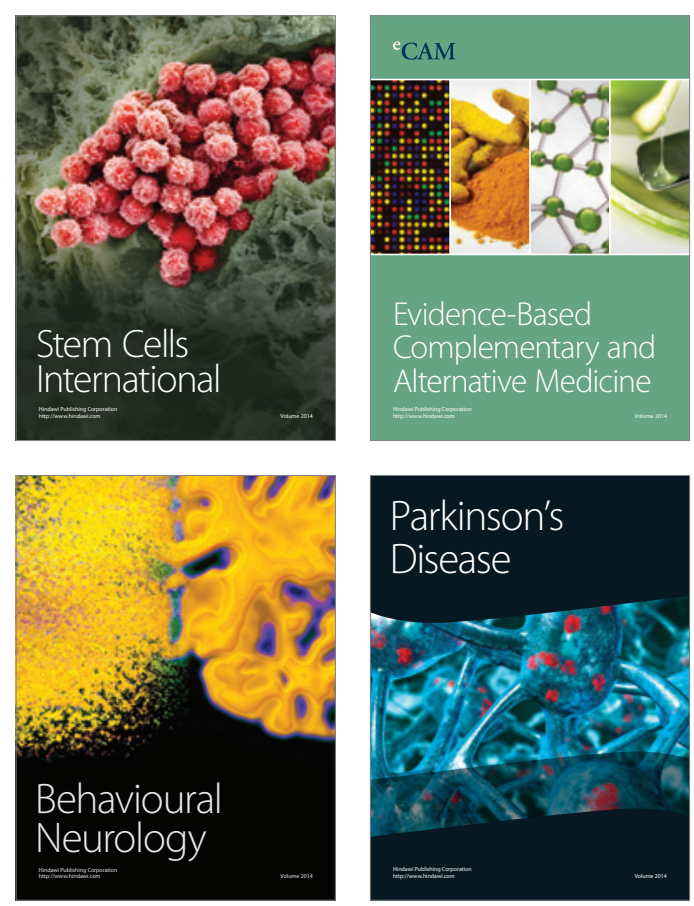

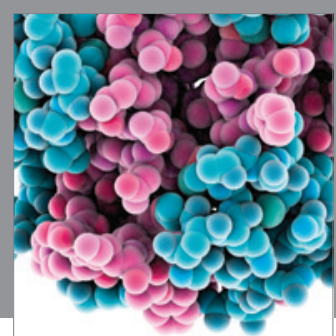

Journal of
Diabetes Research

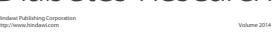

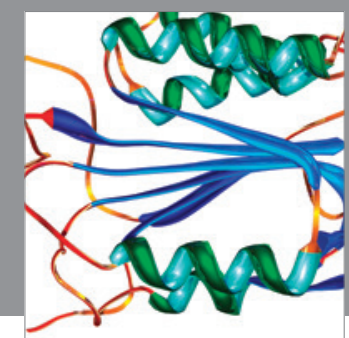

Disease Markers
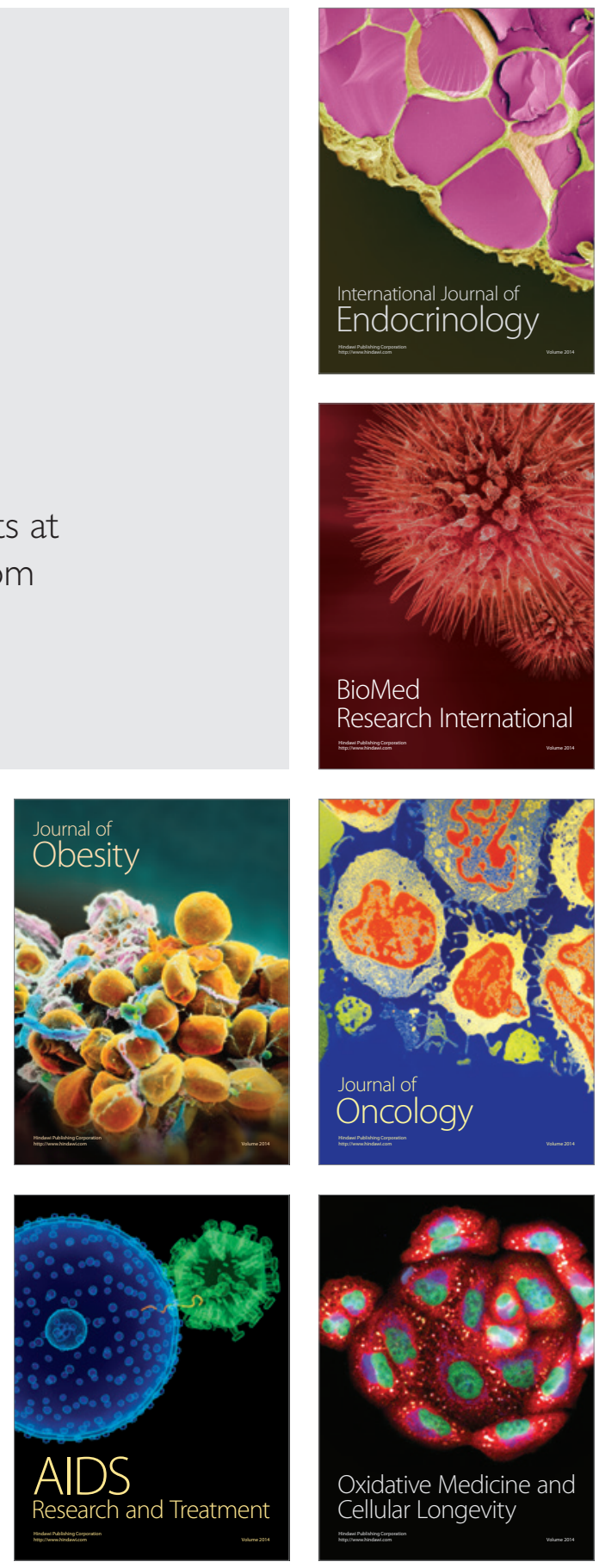